\title{
Lesson of the month 2: Cauda equina in Cushing's syndrome
}

\author{
Authors: Douglas Maslin, ${ }^{A}$ Ioannis Gounaris, ${ }^{B}$ Kenrick $\mathrm{Ng}^{C}$ and Pippa Corrie ${ }^{D}$
}

\begin{abstract}
We present the case of a 34-year old woman who initially presented with obesity and back pain. She was eventually diagnosed with Cushing's syndrome secondary to an adrenocortical carcinoma that had metastasised to her spine, causing cauda equina compression. The delays in reaching the correct diagnosis caused significant morbidity and exemplify the pitfalls of premature closing, a common cognitive error in diagnostic reasoning.
\end{abstract}

KEYWORDS: Cushing's syndrome, adrenocortical carcinoma, cauda equina, back pain, premature closing

\section{Case report}

A 34-year-old woman presented to the emergency department with severe central lower back pain and paraplegia. The back pain had started after her bed collapsed two months previously and had since been steadily worsening. She had recently visited her general practitioner who diagnosed mechanical back pain secondary to obesity and prescribed simple analgesics. She had visited a chiropractor four days previously and also attended the emergency department at another hospital two days before. On that occasion, examination identified tender lower thoracic and lumbar vertebrae and paraspinal muscles, but a full neurological examination was not carried out ('pain limited examination'). The diagnosis of musculoskeletal back pain secondary to morbid obesity was made and the patient discharged with a plan for outpatient lumbar radiographs. On presentation to our institution, she had been unable to walk for six days.

The patient reported significant weight gain over the preceding 18 months (from clothing size 16 to 28 ) and now weighed $172 \mathrm{~kg}$. She reported low mood and amenorrhoea over the last 12 months. She had been prescribed sertraline for depression and furosemide for lower limb oedema. Blood tests performed a few months before had shown low folliclestimulating hormone $(<0.2 \mathrm{U} / \mathrm{L}$; normal range, 2.9-8.4 U/L) and luteinising hormone $(<0.2 \mathrm{U} / \mathrm{L}$; normal range, $1.3-8.4 \mathrm{U} / \mathrm{L})$ with mildly elevated thyroid stimulating hormone $(6.44 \mathrm{mU} / \mathrm{L}$;

Authors: A academic CMT, Oncology, Addenbrooke's Hospital, Cambridge, UK; ${ }^{\mathrm{B}}$ consultant oncologist, Medical Oncology, Queen Elizabeth Hospital, Birmingham, UK; ${ }^{C} \mathrm{CMT}$, Oncology, Royal Free Hospital, London, UK; ' Consultant oncologist, Medical Oncology, Addenbrooke's Hospital, Cambridge, UK normal range, $0.35-5.50 \mathrm{mU} / \mathrm{L}$ ), but these findings were not investigated further.

On examination, there was evidence of centripetal obesity, proximal muscle wasting with a prominent interscapular fat pad and hirsutism. Her blood pressure was 144/64 mmHg, pulse rate $80 \mathrm{bpm}$, respiratory rate 16 breaths/min and oxygen saturations $98 \%$ on air. Full neurological examination revealed normal cranial nerves; bilateral proximal myopathy in the upper limbs (4/5), decreased power throughout the lower limbs (3/5) with normal sensation, including perineal sensation, and symmetrical $2+$ knee reflexes with downgoing planters bilaterally. She was unable to stand.

Blood tests revealed haemoglobin $130 \mathrm{~g} / \mathrm{L}$ (normal range 115-160), mean corpuscular volume $60 \mathrm{fL}$ (normal range 80-100), Na 138 mmol/L (normal range 133-146), K 4.1 $\mathrm{mmol} / \mathrm{L}$ (normal range 3.5-5.3) and CCa $2.70 \mathrm{mmol} / \mathrm{L}$ (normal range 2.20-2.60). An urgent magnetic resonance image of the whole spine showed extensive vertebral bony metastases with fracture at L2 causing cauda equina compression (Fig 1). Whole body computerised tomography scan revealed a large

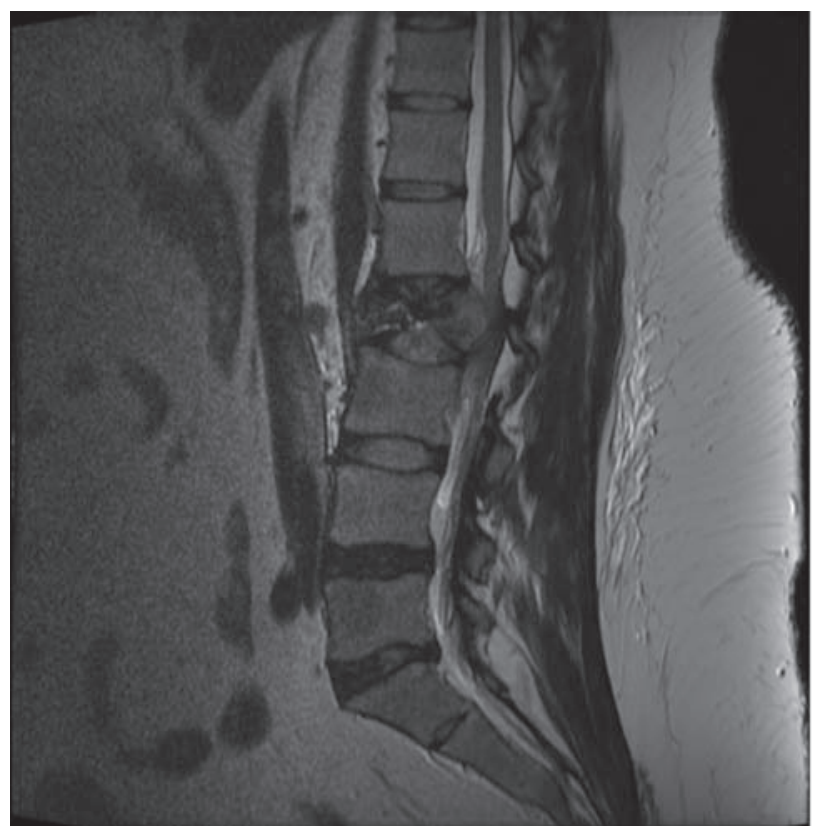

Fig. 1 Sagittal T2 magnetic resonance imaging spine. There is loss of vertebral height of $\mathrm{L} 2$ with retropulsion of the posterior wall into the spinal canal causing compression. Focal areas of low intensity were apparent within T12, L1, L3 and L5 vertebral bodies on T1 imaging. 


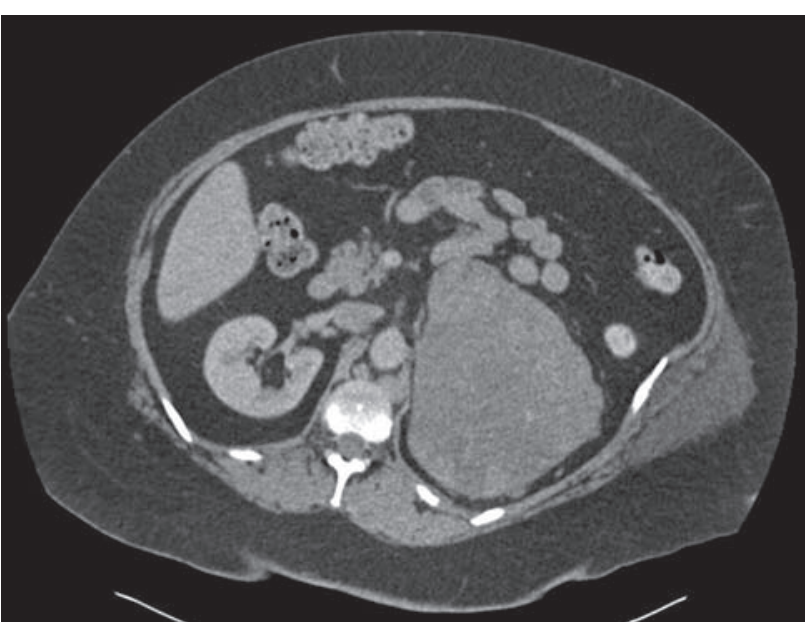

Fig. 2 Computerised tomography chest-abdomen-pelvis. There is a large left adrenal mass measuring $16 \times 13 \times 13 \mathrm{~cm}$; and also significant paraaortic lymphadenopathy. This study also revealed thrombus within the left renal vein; bilateral pulmonary emboli; and multiple bony deposits.

left adrenal mass measuring 16x13x13 cm (Fig 2), paraaortic lymphadenopathy, bone and lung metastases and bilateral pulmonary emboli. Random serum cortisol was elevated (1,019 nmol/L) and was not suppressed on low-dose dexamethasone testing $(979 \mathrm{nmol} / \mathrm{L}$, normal $<50 \mathrm{nmol} / \mathrm{L})$, confirming the clinical diagnosis of Cushing's syndrome. Of note, urinary metanephines were not elevated, but many steroids frequently associated with hypercortisolism, including dehydroepiandrosterone sulphate with a value of $41.0 \mu \mathrm{mol} / \mathrm{L}$ (1.5-11.6), were markedly so. These findings confirmed a diagnosis of a cortisol-secreting primary adrenocortical carcinoma (ACC), so tissue biopsy was not pursued. Palliative chemotherapy was considered but the patient's liver function deteriorated; she was transferred to hospice care and died 7 weeks after presentation.

\section{Discussion}

Cushing's syndrome results from chronic exposure to excess cortisol. When hypercortisolism is severe and prolonged, the diagnosis is straightforward. However, in many cases, as exemplified by our patient, the diagnosis can be delayed as the connections among the developing symptoms and signs are not appreciated, and a unifying diagnosis is not pursued. Such patients are often treated for hypertension in the community, their obesity is attributed to lifestyle, and they are frequently referred to subspecialists such as gynaecologists (oligomenorrhoea and infertility), dermatologists (acne, striae and red facial skin) or orthopaedics/rheumatology (low mineral density and fragility fractures) before a diagnosis is made. ${ }^{1}$

Adrenal adenomas and carcinomas constitute the second most frequent cause of endogenous (not due to corticosteroid therapy) Cushing's syndrome, accounting for $15-30 \%$ of cases. ${ }^{2}$ In total, 30-40\% of adrenal cortical carcinomas (ACC) exhibit clinical signs of cortisol excess at presentation. ${ }^{3}$ Nevertheless, both endogenous Cushing's syndrome and ACC are rare diseases with an incidence of $<5$ and $<2$ per million population respectively. ${ }^{2,3}$ The clinical presentation of metastatic ACC is variable but prognosis uniformly poor.

Over the course of two months, this patient presented to multiple clinicians with back pain which was twice diagnosed as musculoskeletal secondary to obesity. While the diagnosis is apparent in retrospect, it should be kept in mind that back pain, obesity and depression are all common scenarios in clinical practice and frequently coexist. Acute lower back pain is responsible for a substantial number of primary and secondary care attendances (with a lifetime prevalence of up to 90\%), and approximately $95 \%$ are due to benign causes. ${ }^{4}$ However, a benign cause should never be assumed prior to assessment, even in younger patients, as the consequences of acute spinal cord and cauda equina compression can be devastating. Incidences range from 1 to 8 in 100,000; and are most commonly due to lumbar disc herniation, spinal stenosis and tumours. ${ }^{5}$ Additionally, a meta-analysis of cauda equina syndrome has shown the importance of promptly treating compression within 48 hours of the onset of symptoms - in terms of motor, sensory and autonomic outcomes. 'Red-flag' symptoms and signs are commonly used as markers of possible serious underlying spinal pathology requiring further investigation ${ }^{8}$. Of those, this patient exhibited prolonged duration of pain, progressing unremitting pain, history of trauma and lower limb neurological signs. ${ }^{7}$

The patient suffered considerable delays in making two crucial diagnoses: Cushing's syndrome and cauda equina syndrome. The delays had a significant effect on her quality of life and arguably contributed to the poor outcome. Diagnostic errors and delays are more often secondary to cognitive rather than system-related factors. ${ }^{8,9}$ Faulty synthesis and premature closure, ie the failure to consider alternative diagnoses after an initial diagnosis is reached, underlie most cognitive errors and contributed to the delays in the case of our patient. Her initial weight gain was attributed to depression and then all subsequent symptoms and syndromes, including the back pain, were explained as sequelae of morbid obesity. Had that premature diagnostic closure not occurred, an endocrine cause of her symptoms would have been evident much sooner leading to an earlier diagnosis of the ACC as the underlying cause. Furthermore, notwithstanding the missed diagnosis of Cushing's syndrome, the same cognitive process led to underinvestigation of the back pain resulting in her presentation with paraplegia; it may have been reasonable to attribute it to mechanical causes initially, but the continuing progression of her symptoms should have prompted further assessment earlier.

\section{Key lessons}

> In the assessment of back pain, a benign cause should never be assumed (regardless of age) - it is thus essential to exclude any 'red flag'-features and to perform a neurological assessment.

> Avoiding premature diagnostic closure and reformulating the differential diagnosis as evidence accumulates are fundamental aspects of good clinical practice that should enable prompter diagnosis of rare but well described and known clinical conditions. Here, the clarity of the diagnosis with the benefit of retrospection enforces this point. 


\section{References}

1 Nieman LK. Cushing's syndrome: update on signs, symptoms and biochemical screening. Eur J Endocrinol 2015;173:M33-8.

2 Lacroix A, Feelders RA, Stratakis CA, Nieman LK. Cushing's syndrome. Lancet 2015;386:913-27.

3 Veytsman I, Nieman L, Fojo T. Management of endocrine manifestations and the use of mitotane as a chemotherapeutic agent for adrenocortical carcinoma. J Clin Oncol 2009;27:4619-29.

4 Jarvik JG, Deyo RA. Diagnostic evaluation of low back pain with emphasis on imaging. Ann Intern Med 2002;137:586-97.

5 Ma B, Wu H, Jia LS, Yuan W, Shi GD, Shi JG. Cauda equine syndrome: a review of clinical progress. Chin Med J (Engl) 2009;122:1214-22.

6 Ahn UM, Ahn NU, Buchowski JM et al. Cauda equina syndrome secondary to lumbar disc herniation: a meta-analysis of surgical outcomes. Spine (Phila Pa 1976) 2000;25:1515-22.
7 van Tulder M, Becker A, Bekkering T et al. Chapter 3 European guidelines for the management of acute nonspecific low back pain in primary care. Eur Spine J 2006;15(Suppl 2):S169-91.

8 Graber ML, Franklin N, Gordon R. Diagnostic error in internal medicine. Arch Intern Med 2005;165:1493-9.

9 Kuhn GJ. Diagnostic errors. Acad Emerg Med 2002;9:740-50.

Address for correspondence: Dr D Maslin, Cambridge Academic Training Office, University of Cambridge, School of Clinical Medicine, Box 111, Cambridge Biomedical Campus,

Cambridge CB2 0SP, UK.

Email: dlm37@cam.ac.uk
Assessing trainees in the workplace An e-learning module for secondary care doctors

Three hours of CPD-approved interactive learning covering:

$>$ feedback

> supervised learning events (SLES)

$>$ workplace-based assessments (WPBAs)

$>$ the role of the Annual Review of Competence Progression (ARCP).

For more information please visit: www.rcplondon.ac.uk/elearning

\section{Royal College} of Physicians

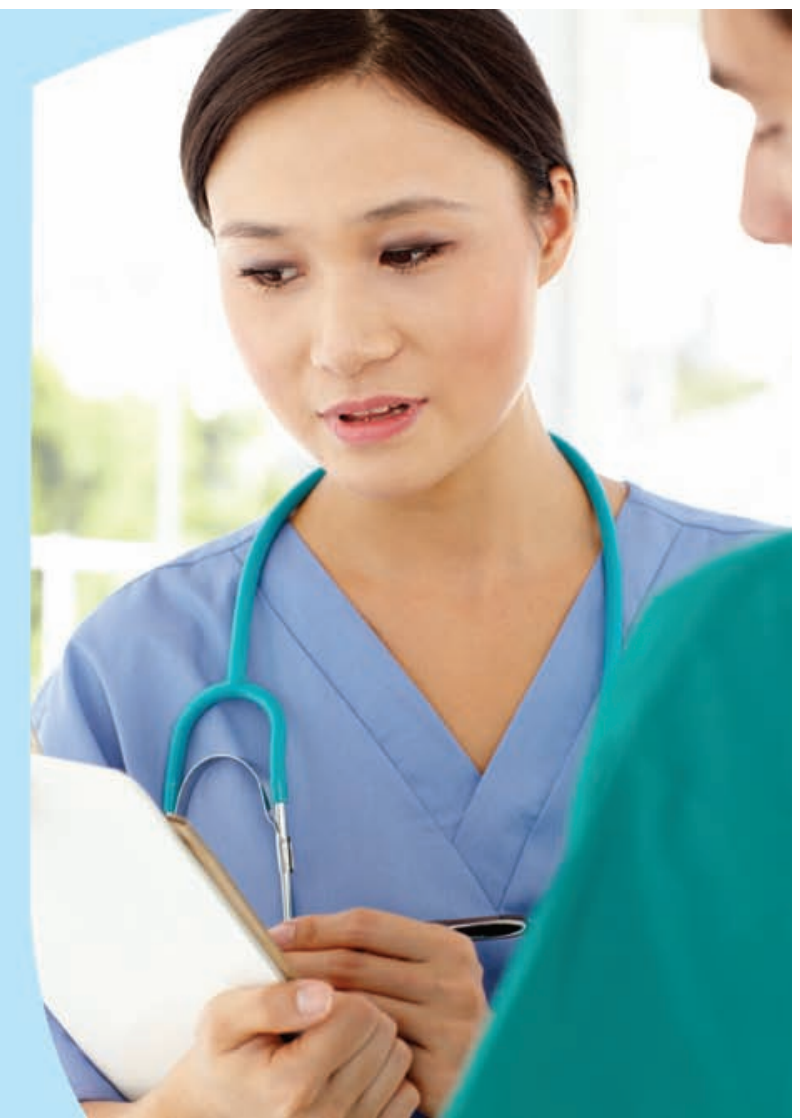

